\title{
Intensity of Effort in Contests with Few or Many Winners
}

\author{
Dimitry Rtischev ${ }^{1)}$
}

\begin{abstract}
The paper applies multi-prize contest theory to explore the connection between scarcity of prizes and intensity of competition. Equilibrium levels of effort are compared across contests that differ in the number of prizes and in which the utility of winning or losing is influenced by the relative numbers of winners and losers. Application to the study of socioeconomic competition is discussed.
\end{abstract}

Keywords: contest theory, socioeconomic competition, psychosocial utility

JEL classification: C72, D91, D11

\section{Introduction}

The goal of this paper is to theoretically explore the connection between the relative scarcity of prizes and the intensity of competition, especially in the context of socioeconomic competition.

Socioeconomic competition for schools, careers, workplaces, neighborhoods, and other sociallyscarce goods is an important component of the economy and determinant of welfare. (Hirsch 1977) The specifics of socioeconomic competition - what is contested and how people compete - are contingent on the institutional context and therefore differ considerably across societies. In some cases socioeconomic competition is more intense and expensive than in others. There are also structural differences in terms of the relative scarcity of the desirable or "winning" outcomes vis-à-vis the less-desirable "losing" outcomes. On the one extreme are competitions resembling races or championships that award only a few highly-valued prizes; on the other are competitions resembling musical chairs in the sense that prizes are plentiful but modest and losing is rare but stigmatizing. Actual cases of socioeconomic competition are situated on the spectrum between these extremes.

The paper focuses on micro-strategic models of allocation in which agents exert costly effort in competition for scarce goods, thereby dissipating some of the value of the goods. Specifically, applying multi-prize contest theory, we vary the number of prizes and document how the degree of scarcity relates to the amount of effort and dissipation in equilibrium. Since winning a rare prize carries greater social and psychological consequences than winning one of many such prizes, in Section 2 we augment agents' utility with a psychosocial component and study how such additional utility influences agents' effort and the resulting value dissipation. To endogenize prize values and get closer to the context of socioeconomic competition, in Section 3 we define and study a competitive allocation process that lets

1) Faculty of Economics, Gakushuin University, Tokyo, Japan. 
all agents first compete in a contest for a limited number of priority tickets and then lets the winners choose the best goods first, leaving the worse goods for the losers. In Section 4 we analyze competition among heterogeneous agents.

\section{Contest to allocate $m$ identical prizes to $n$ identical agents}

Suppose there are $n$ identical risk-neutral agents and $m<n$ identical prizes, each of which has intrinsic value $v$ to any agent who gets one. The prizes are allocated via a contest in which each agent makes an effort $e_{i}$ and can win at most one prize. If $m$ is small, the contest is like a race that awards medals to the fastest few; if $m$ is closer to $n$, the contest is like musical chairs. Specifically, the utility obtained by an agent $i$ if it wins a prize is

$$
u_{i}^{\text {win }}=v-e_{i}+w(n, m)
$$

and the utility it obtains if it doesn't win is

$$
u_{i}^{\text {lose }}=-e_{i}-l(n, m)
$$

These expressions indicate that in addition to getting or not getting the intrinsic value of the prize, agents also benefit or suffer from psychosocial consequences. For reasons to be discussed in the following section, we will assume that a winner's psychosocial utility $w(n, m)$ is weakly decreasing in $m$ whereas a loser's psychosocial disutility $l(n, m)$ is weakly increasing in $m$.

Let us denote the probability that agent $i$ who makes effort $e_{i}$ wins a prize in the contest by $p_{i}\left(e_{i}, e_{-i}\right)$, which is a contest success function that is increasing in the agent's own effort $e_{i}$ and decreasing in the sum of all other agents' efforts

$$
e_{-i} \equiv \sum_{j \neq i} e_{j}
$$

Each agent chooses effort $e_{i}$ so as to maximize its expected utility given by

$$
E\left[u_{i} \mid e_{i}, e_{-i}\right]=p_{i}\left(e_{i}, e_{-i}\right)(v+w(n, m))-\left(1-p_{i}\left(e_{i}, e_{-i}\right)\right) l(n, m)-e_{i}
$$

Re-arranging this as

$$
E\left[u_{i} \mid e_{i}, e_{-i}\right]=p_{i}\left(e_{i}, e_{-i}\right)(v+w(n, m)+l(n, m))-e_{i}-l(n, m)
$$

reveals that expanding the contest model to include psychosocial utility changes the agents' utilitymaximization problem in a very simple way, namely the psychosocial utility and disutility are both added to the intrinsic prize value $v$. Since the last term is independent of effort, it does not enter the 
maximization problem.

Following Clark and Riis $(2006)^{2)}$, we assume the following underlying process for allocating prizes: first all agents irreversibly invest their efforts $\left\{e_{i}\right\}$; then $m$ independent random draws are used to allocate the $m$ prizes one after another in a way that excludes agents who already won a prize from participating in subsequent draws. On any given draw, the probability that an agent $i$ participating in that draw wins a prize is given by the Tullock ratio of the agent's effort to the sum of the efforts of all the agents who participate in that draw. As proven by Clark and Riis (2006, equation 8), in the symmetric Nash equilibrium each agent makes the effort:

$$
e^{*}(n, m)=\frac{1}{n}\left[\frac{m(n-1)}{n}-\sum_{j=1}^{m-1} \frac{m-j}{n-j}\right](v+w(n, m)+l(n, m)), \quad 1<m<n
$$

The fraction of aggregate prize value $m v$ that is dissipated by all the agents' efforts is given by

$$
\rho(n, m)=\frac{n e^{*}}{m v}
$$

\subsection{Baseline contest}

As a baseline, let us first examine effort and dissipation without psychosocial utility. We set $w(n, m)=$ $l(n, m)=0$ and examine (6) and (7) as a function of $m$. Although we cannot obtain a closed-form solution, numerical analysis reveals that equilibrium effort (6) has a unique maximum of $0.367 \mathrm{v}$ at $m=$ $0.632 n$. That is, individual effort reaches its peak of about $37 \%$ of prize value when there are enough prizes for about $63 \%$ of the population. The dissipation fraction (7) is monotonically decreasing from near 1 to near 0 as $m$ increases from 2 to $n-1$. Thus, as the prizes become rare and the population large, the agents collectively use up all of the aggregate prize value.

Equilibrium effort (6) and dissipation ratio (7) are plotted as a function of the number of prizes in Figures 1 and 2, respectively. Given any two cases we cannot immediately say whether the case closer to musical chairs extreme has more intense competition than the case closer to the race extreme. For example, in a contest with 100 agents and prizes of value 1, each agent invests 0.18 if there are 20 prizes, 0.36 if there are 60 prizes, and 0.15 if there are 95 prizes. Viewed in aggregate, however, the effect of prize scarcity is monotonic: when there are 20 prizes the agents altogether spend $89 \%$ of the value of all the prizes on competing for them, when there are 60 prizes they spend $61 \%$, and when there are 95 prizes they spend only $15 \%$. Thus, whereas in aggregate a contest closer to the race extreme dissipates a higher fraction of prize value than does a contest closer to the musical chairs extreme, there are contests closer to the musical chairs extreme in which each agent spends more on competition than in other contests closer to the race extreme.

2) See also Konrad (2009, Section 4.2) for an overview of multi-prize contest theory. 


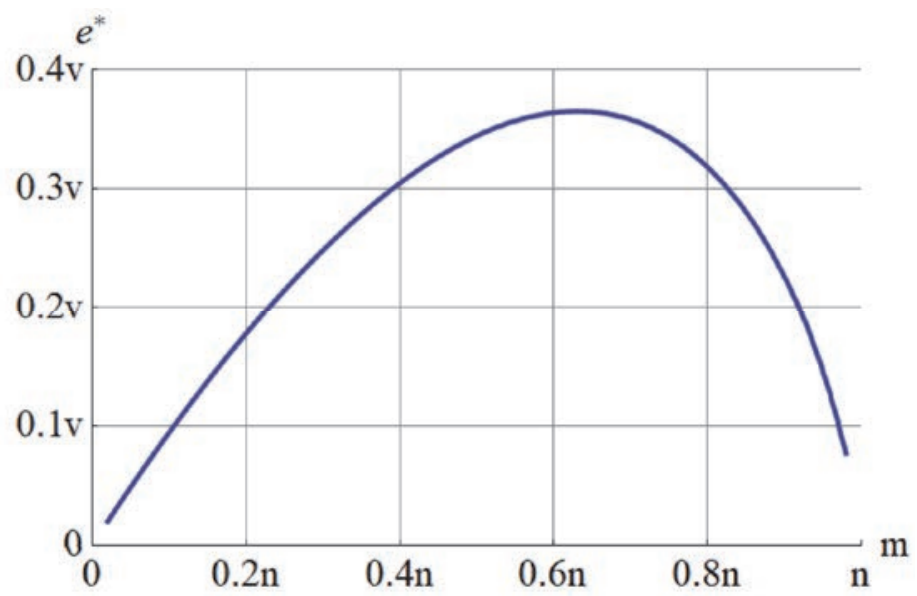

Figure 1. Individual competitive effort by each agent in equilibrium without psychosocial utility.

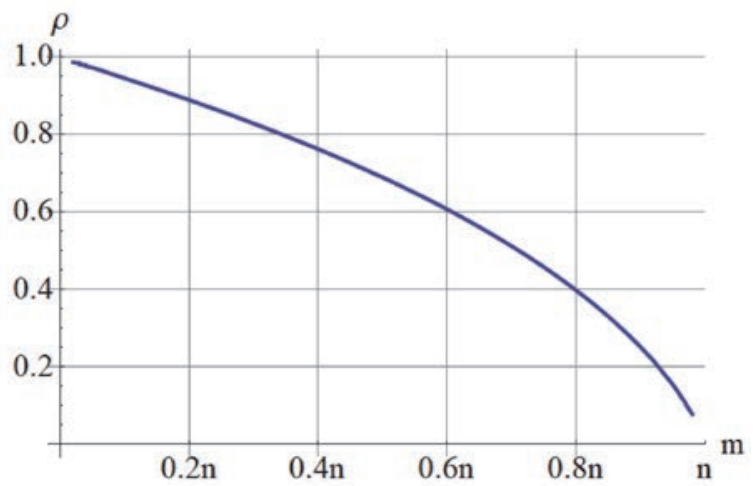

Figure 2. Fraction of total prize value dissipated in equilibrium without psychosocial utility.

\subsection{Psychosocial utility}

In a game of musical chairs, the loser left standing is isolated and embarrassed but each sitting winner doesn't feel very special. Feelings after a race are quite different: the fastest three are honored with medals atop a pedestal, but the also-ran are not isolated and shamed. Although both races and musical chairs are forms of competition to allocate scarce resources, they are very different in terms of their social and psychological consequences for the winners and losers. Whether a given form of competition creates proud heroes or shameful failures seems to depend on the relative numbers of winners and losers. 
Those who win and those who lose are both better off when prizes are rare. ${ }^{3)}$

In light of these considerations, let us define psychosocial utility in terms of the fraction of the number of winners $m$ to the number of losers $n-m$ and scale them with constants $W>0$ and $L>0$ as follows:

$$
\begin{aligned}
& w(n, m)=\frac{n-m}{m} W \\
& l(n, m)=\frac{m}{n-m} L
\end{aligned}
$$

Under these definitions, the winner's utility rises sharply as prizes become rare and the loser's disutility rises sharply as prizes become very common. (Figure 3)

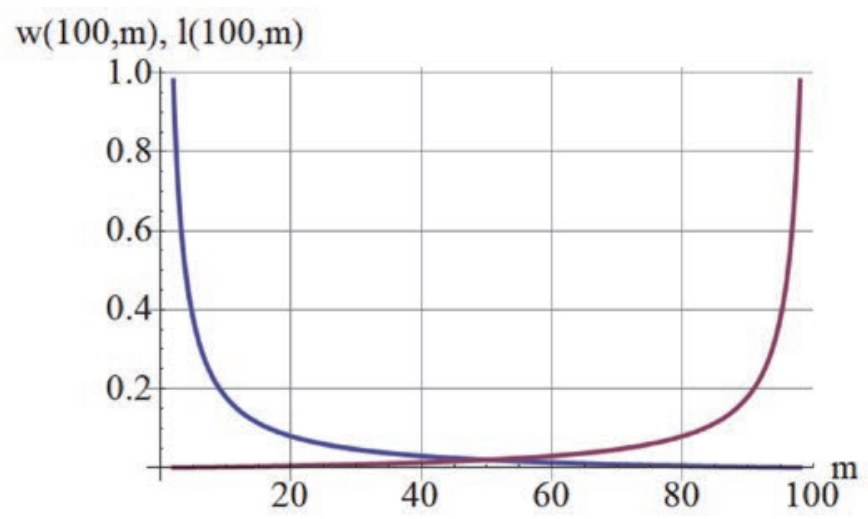

Figure 3. Psychosocial utility of winners (falling curve) and disutility of losers (rising curve) as a function of number of prizes. This is a plot of (8) and (9) with $n=100$ and $W=L=0.02$.

Figure 4 plots equilibrium effort (6) as a function of the number of prizes. The plot shows that adding psychosocial utility that is symmetrical with respect to winning or losing ${ }^{4}$ (i.e., $W=L$ ) raises individual competitive effort on the musical chairs side of the spectrum more than on the race side. With sufficiently strong psychosocial effects, the shape of the curve is altered such that musical chairs elicit more competitive effort than races on the entire spectrum. Moreover, musical chairs can even motivate agents to put in effort that far exceeds the value of the prize, as shown by the portions of the curves in Figure 4 lying above the $e^{*}=1$ line.

3) Proverbs such as "misery loves company" and "to honor all is to honor none" (Moliere, The Misanthrope, 1666) corroborate the idea that losers find comfort in numbers whereas winners find pride in rarity. Moreover, trying to win a rare prize corresponds to "approach" and "challenge" whereas trying to avoid being a rare loser corresponds to "avoidance" and "threat" in the psychological research on approach vs. avoidance motivation (Elliot 2008) and in the biopsychosocial model of challenge and threat (Blascovich 2008).

4) Although we confine our analysis to the symmetric case, it is conceivable that the psychosocial disutility of losers exceeds the psychosocial utility of winners, analogously to loss-aversion in prospect theory. 


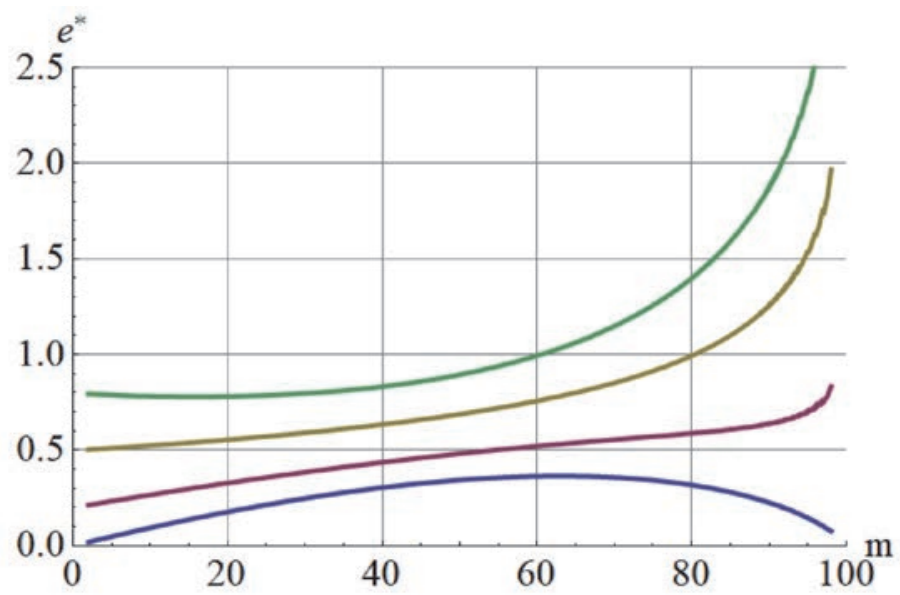

Figure 4. Individual competitive effort by each of 100 agents with psychosocial effects as defined in (8) and (9). The four curves show equilibrium effort (6) with parameter values $(W, L)=(0,0),(0.2,0.2),(0.5,0.5),(0.8,0.8)$ from bottom to top, $n=100$, $v=1$.

Aggregate dissipation is also higher when psychosocial utility is added. As Figure 5 shows, agents may collectively dissipate many times the value of all the prizes combined. Races are more wasteful than musical chairs, as was the case without psychosocial utility, but in the presence of sufficiently large psychosocial utility the dissipation contour also rises towards the musical chairs extreme.

\section{Winners-choose-first contest to allocate goods of varying quality to all agents}

To take a step closer to modeling socioeconomic competition and endogenize prize values, we next study an allocation process in which $n$ identical risk-neutral agents compete for $n$ goods that vary in quality. We assume that there are enough goods for all agents, but there is competition to determine who gets to choose first. Specifically, the process we study proceeds in two stages as follows ${ }^{5)}$ :

1. Competition Stage: Each agent $i$ invests effort $e_{i}$ in a contest to win one of the $m<n$ identical priority tickets.

2. Allocation Stage: First, the $m$ best goods are allocated randomly to the $m$ agents with tickets, one good per agent. Then, the $n-m$ worst goods are allocated randomly to the $n-m$ agents without tickets.

There is consensus among agents about the goods: the value of a good $i$ to any agent is $v_{i}$. The value of the goods is distributed on $[0, V]$ according to a cumulative function $F(v)$ which indicates the number

5) This process loosely resembles competition for education followed by the allocation of the better jobs to the better-educated. 

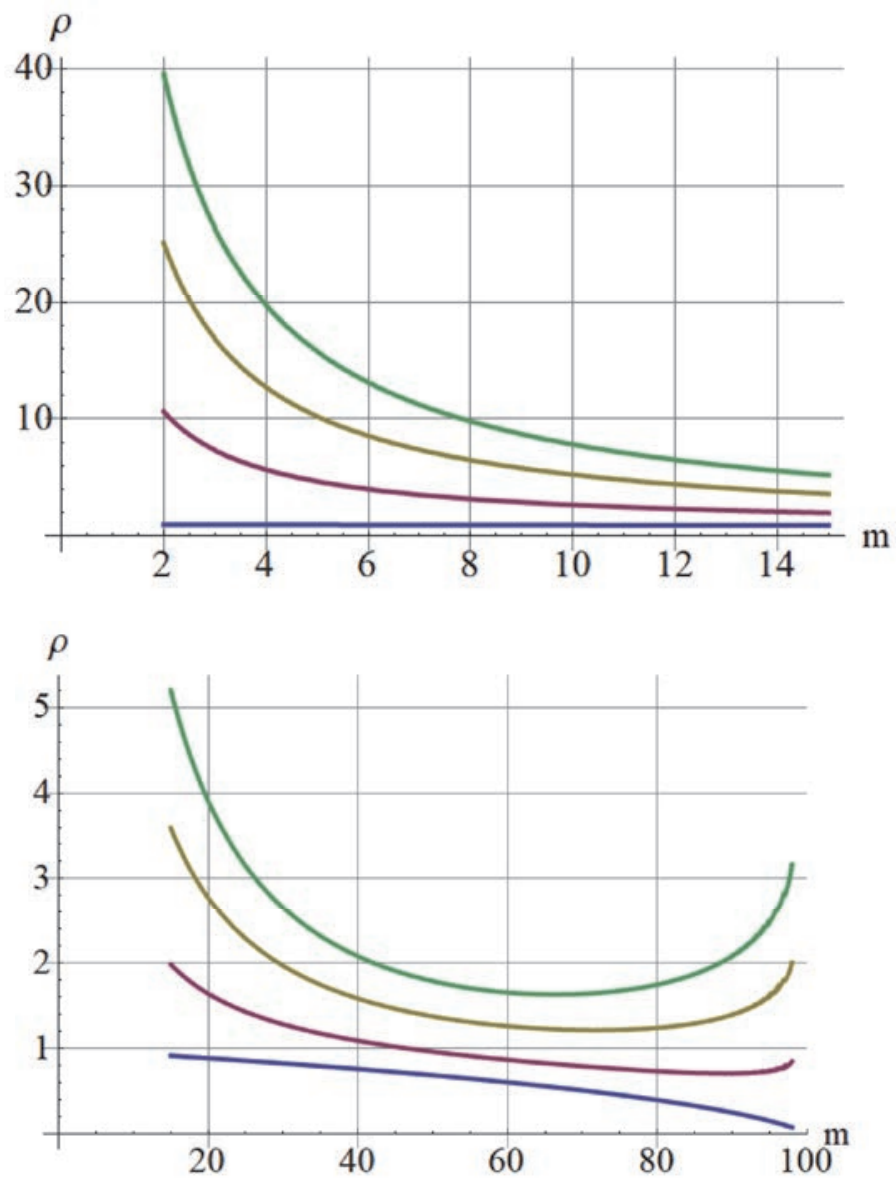

Figure 5. Fraction of total prize value dissipated in equilibrium by a population of 100 agents with psychosocial effects per (8) and (9). The four curves show aggregate dissipation $(7)$ with parameter values $(W, L)=(0,0),(0.2,0.2),(0.5,0.5),(0.8,0.8)$ from bottom to top, $n=100, v=1$. The graph is split for clarity: the top part shows the range $m=2$ to 15 and the bottom part shows $m=15$ to 98 .

of goods of value $v$ or less. The corresponding density function ${ }^{6)} f(v)$ has support on $[0, v]$, has a unique interior maximum, and is normalized to satisfy

$$
\int_{0}^{V} f(v) d v=n
$$

6) Since there are $n$ discrete goods, it is proper to define $f()$ as a discrete probability mass function. However, for analytical convenience, we will treat $f()$ as a continuous density. 
Let $\theta(\mathrm{n}, \mathrm{m})$ be the value of the $m^{\text {th }}$ best good among $n$ goods, defined as follows:

$$
\theta(\mathrm{n}, \mathrm{m})=F^{-1}(n-m)
$$

The expected payoff to an agent who wins a ticket is the expected value of the best $m$ goods given by

$$
\bar{v}(n, m)=\frac{1}{m} \int_{\theta(n, m)}^{V} v f(v) d v
$$

The expected payoff to an agent who doesn't win a ticket is the expected value of the worst $n-m$ goods given by

$$
\underline{v}(n, m)=\frac{1}{n-m} \int_{0}^{\theta(n, m)} v f(v) d v
$$

The utility obtained by an agent $i$ if it exerts effort $e_{i}$ and wins a priority ticket is

$$
u_{i}^{\text {win }}=\bar{v}(n, m)-e_{i}
$$

and the utility it obtains if it doesn't win a ticket is

$$
u_{i}^{\text {lose }}=\underline{v}(n, m)-e_{i}
$$

As in Section 2, each agent chooses effort $e_{i}$ so as to maximize its expected utility given by

$$
E\left[u_{i} \mid e_{i}, e_{-i}\right]=p_{i}\left(e_{i}, e_{-i}\right) \bar{v}(n, m)+\left(1-p_{i}\left(e_{i}, e_{-i}\right)\right) \underline{v}(n, m)-e_{i}
$$

Re-arranging this expression gives:

$$
E\left[u_{i} \mid e_{i}, e_{-i}\right]=p_{i}\left(e_{i}, e_{-i}\right)(\bar{v}(n, m)-\underline{v}(n, m))-e_{i}+\underline{v}(n, m)
$$

Since the last term is constant with respect to effort, the agents' maximization problem depends solely on the difference between the expected value of the winners' goods and the losers' goods. Re-writing equilibrium effort (6) for this case gives:

$$
e^{*}(n, m)=\frac{1}{n}\left[\frac{m(n-1)}{n}-\sum_{j=1}^{m-1} \frac{m-j}{n-j}\right](\bar{v}(n, m)-\underline{v}(n, m)), 1<m<n
$$


The fraction of the aggregate value of the goods that is dissipated by the total of the agents' efforts is given by

$$
\rho(n, m)=\frac{n e^{*}(n, m)}{\int_{0}^{V} v f(v) d v}
$$

\subsection{Triangular distribution of goods}

The triangular distribution (Figure 6) offers a tractable approximation to the normal distribution that we shall consider in the following subsection.

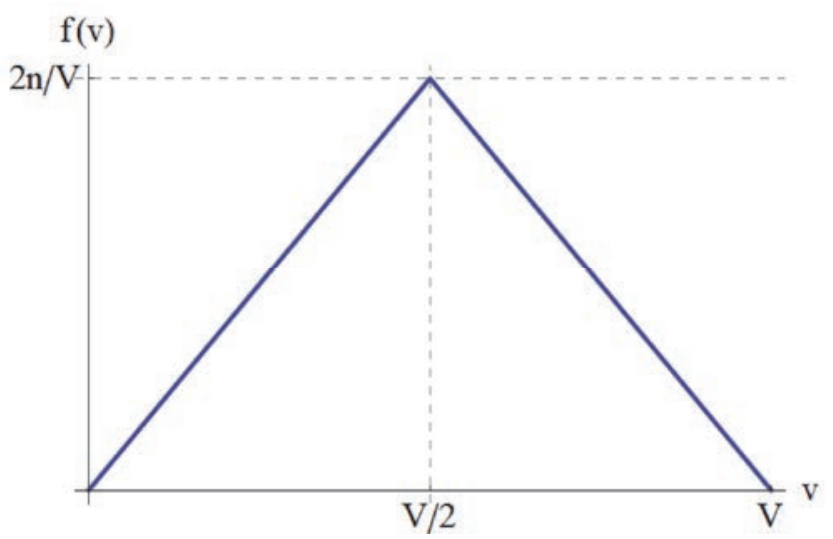

Figure 6. Triangular distribution of good values.

For the case of the triangular distribution, the expected value of the best $m$ goods and the worst $n-m$ goods are given by the following expressions and plotted in Figure 7.

$$
\begin{aligned}
& \bar{v}(n, m)=\left\{\begin{array}{cc}
\frac{V}{3}\left[3-\sqrt{\frac{2 m}{n}}\right], & m<\frac{n}{2} \\
\frac{n V}{6 m}\left[3-2 \sqrt{2}\left(1-\frac{m}{n}\right)^{3 / 2}\right], & m>\frac{n}{2}
\end{array}\right. \\
& \underline{v}(n, m)=\left\{\begin{array}{cc}
\frac{V}{6(n-m)}\left[2 m\left(\sqrt{\frac{2 m}{n}}-3\right)+3 n\right], & m<\frac{n}{2} \\
\frac{V}{3} \sqrt{\frac{2(n-m)}{n}}, & m>\frac{n}{2}
\end{array}\right.
\end{aligned}
$$




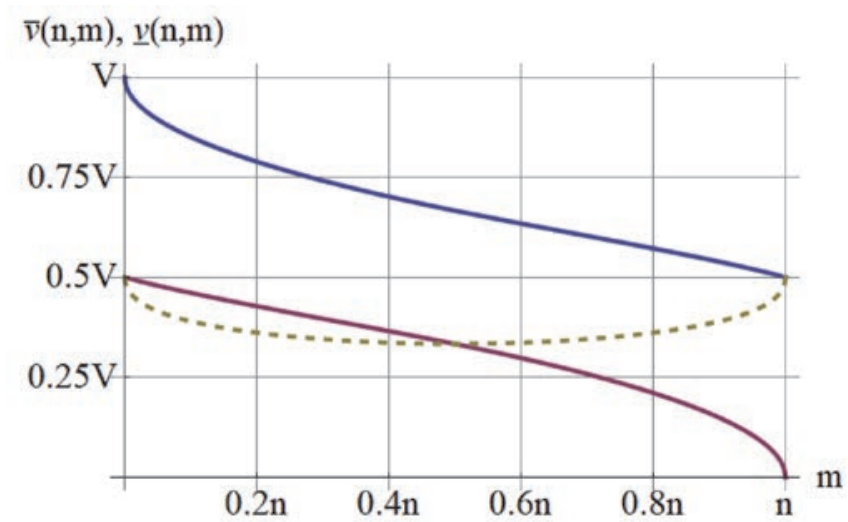

Figure 7. Expected value of the best $m$ goods (eqn. 20, upper curve) and the worst $n-m$ goods (eqn. 21, lower curve), and their difference (dashed curve) as a function of $m$ for the case of the triangular distribution.

According to (18), equilibrium effort depends on the difference in the expected value of the best $m$ goods and the worst $n-m$ goods. Subtracting (21) from (20) yields (22), which is plotted as the dashed curve in Figure 7:

$$
\bar{v}(n, m)-\underline{v}(n, m)= \begin{cases}\frac{n V}{6(n-m)}\left[3-2 \sqrt{\frac{2 m}{n}}\right], & m<\frac{n}{2} \\ \frac{n V}{6 m}\left[3-2 \sqrt{2-\frac{2 m}{n}}\right], & m>\frac{n}{2}\end{cases}
$$

Figure 8 plots the individual equilibrium effort (18) and dissipation ratio (19) as a function of the number of tickets $\mathrm{m}$. Both the effort and the dissipation ratio peak when the number of tickets is about $2 / 3$ of the number of agents. The maximum effort is $V / 8$ and the maximum dissipation ratio is 0.25 . 


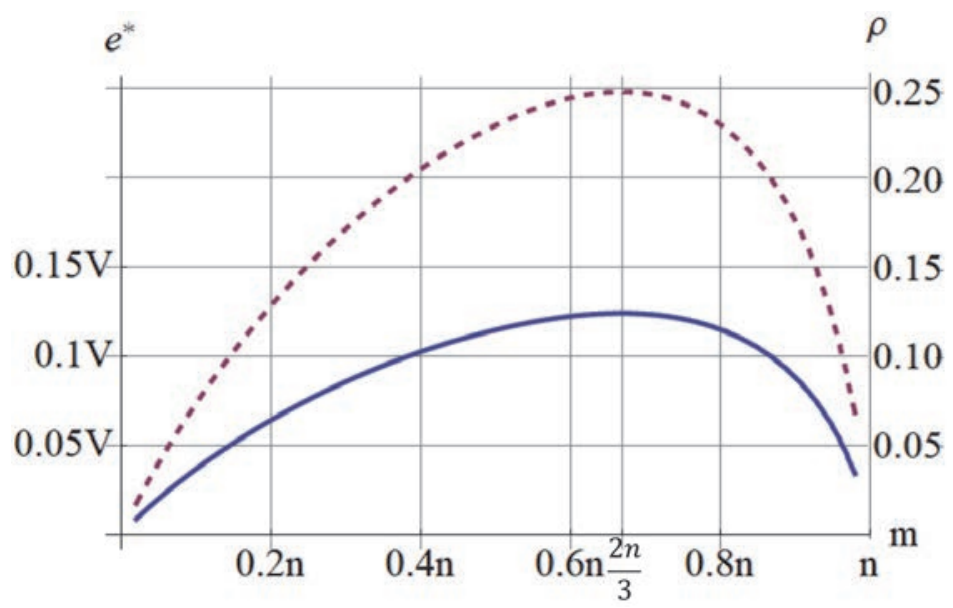

Figure 8. Equilibrium effort (eqn. 18, solid curve, left axis) and dissipation ratio (eqn. 19, dashed curve, right axis) in the contest for priority tickets given the triangular distribution of good values. Effort and dissipation peak at $0.125 \mathrm{~V}$ and 0.25 , respectively, when the number of tickets is $2 / 3$ of the number of agents.

\subsection{Truncated normal distribution of goods}

In this Section we examine equilibrium effort (18) and the dissipation factor (19) when the distribution of good values is a truncated normal on $[0, \mathrm{~V}]$ and centered at $\mathrm{V} / 2$ as shown in Figure 9. ${ }^{7)}$ The results are similar to the case of the triangular approximation examined in the previous subsection.

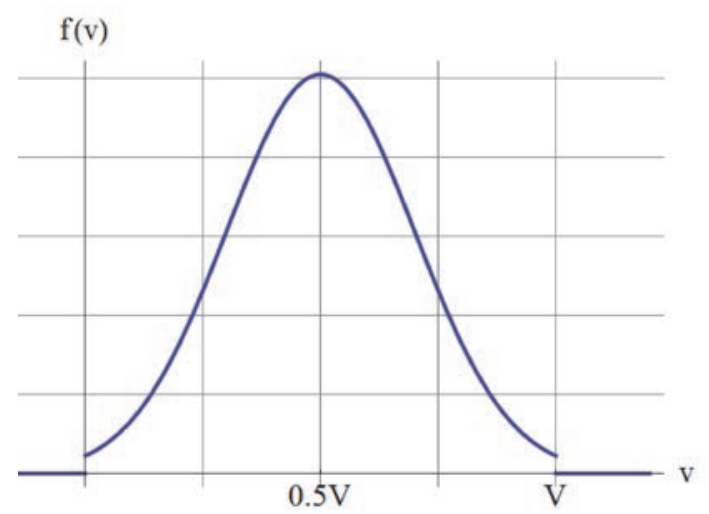

Figure 9. Truncated normal distribution of good values with $V=200$ and standard deviation 40.

7) We truncate the distribution at 0 since we focus on the allocation of positive-valued goods. Truncation at V is not technically necessary but simplifies computation with only a minor effect on results. 
The expected value of the best $m$ goods and the worst $n-m$ goods, and the difference between the two, are plotted in Figure 10.

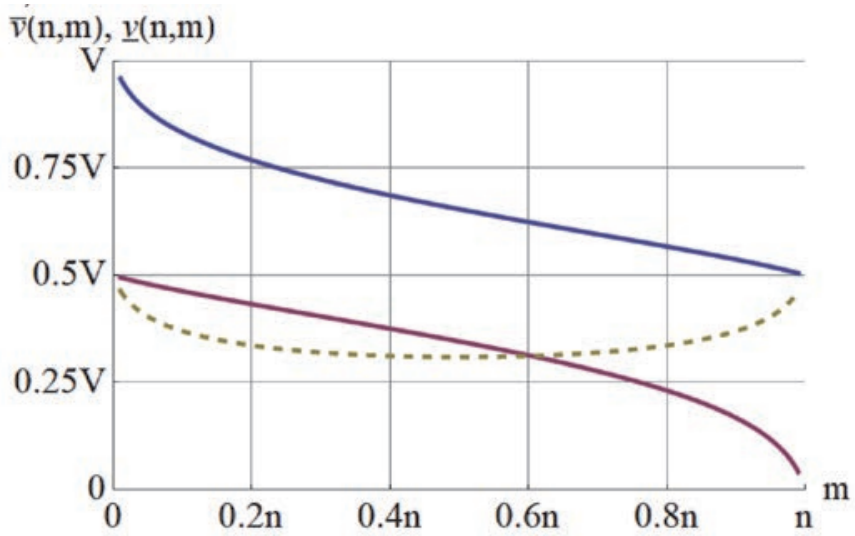

Figure 10. Expected value of the best $m$ goods (eqn. 20, upper curve) and the worst $n-m$ goods (eqn. 21, lower curve), and their difference (dashed curve) as a function of $m$ for the case of the truncated normal distribution.

Figure 11 plots individual equilibrium effort (18) and the aggregate dissipation ratio (19) as a function of the number of tickets $m$. As was the case with the triangular distribution in the previous subsection, both the effort and the dissipation ratio peak when the number of tickets is about $2 / 3$ of the number of agents.

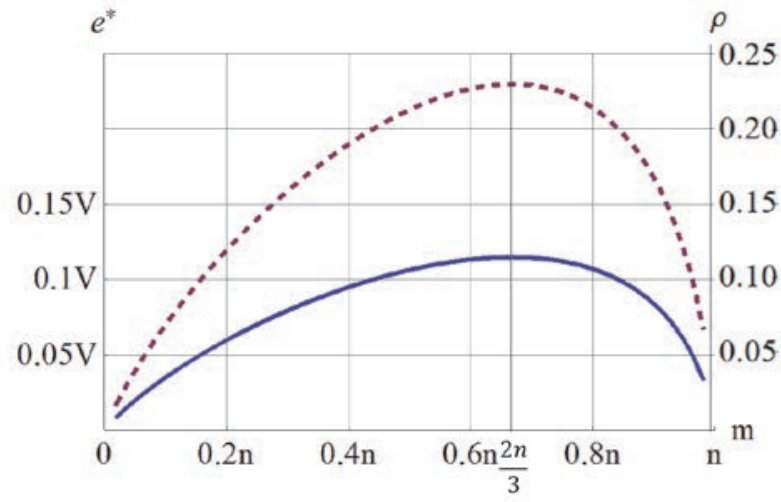

Figure 11. Equilibrium effort (eqn. 18, solid curve, left axis) and dissipation ratio (eqn. 19, dashed curve, right axis) in the contest for priority tickets given the truncated normal distribution of good values with $\mathrm{V}=200$ and standard deviation 40 . Effort and dissipation peak at $0.115 \mathrm{~V}$ and 0.23 , respectively, when the number of tickets is $2 / 3$ of the number of agents. 


\section{Contest to allocate $\mathrm{m}$ identical prizes to $\mathrm{n}$ heterogeneous agents}

So far we have examined allocation of prizes among identical agents. Since people vary in their abilities and preferences, it is important to widen the inquiry to include heterogeneous agents. Although heterogeneous preferences lie beyond the scope of this paper, in this section we will briefly consider agents whose abilities differ in terms a parameter that determines the utility cost of effort. Specifically, in a contest to allocate $m$ identical prizes of value $v$ to $n$ agents, the utility obtained by an agent $i$ if it wins a prize is

$$
u_{i}^{\text {win }}=v-c_{i} e_{i}
$$

and the utility it obtains if it doesn't win is

$$
u_{i}^{\text {lose }}=-c_{i} e_{i}
$$

where $c_{i}>0$ is an exogenously fixed cost parameter specific to agent $i$. We assume that no two agents have the same cost parameter and index the agents in the order of increasing costs: $c_{1}<c_{2}<\ldots<c_{n}$.

Siegel (2009) provides a general characterization of equilibrium payoffs in all-pay multiple-prize contests with heterogenous agents. Applying Siegel's Theorem 1 to our formulation, the expected payoffs of the players in equilibrium are:

$$
E\left[u_{i}\right]=\left\{\begin{array}{cc}
\left(1-\frac{c_{i}}{c_{m+1}}\right) v & \forall i \in\{1, \ldots, m\} \\
0 & \forall i \in\{m+1, \ldots, n\}
\end{array}\right.
$$

Thus, according to (25), in equilibrium only the $m$ most "efficient" agents earn positive expected utility, the amount of which varies directly with the cost advantage over the "marginal" agent $m+1$. The aggregate expected utility of all agents is

$$
U \equiv \sum_{i=1}^{n} E\left[u_{i}\right]=m v-\frac{v}{c_{m+1}} \sum_{i=1}^{m} c_{i}
$$

Since the aggregate value of prizes is $m v$, the fraction of aggregate value dissipated in the contest is

$$
\rho=1-\frac{U}{m v}=\frac{\overline{c_{m}}}{c_{m+1}}
$$

where the numerator is the average cost parameter of the $m$ lowest-cost agents: 


$$
\overline{c_{m}} \equiv \frac{1}{m} \sum_{i=1}^{m} c_{m}
$$

Suppose the agents' cost parameters $c_{i}$ are distributed on $[0, C]$ according to a cumulative distribution function $F(c)$ which indicates the proportion of agents whose cost parameter is $c$ or less. The corresponding density function ${ }^{8)} f(c)$ has support on $[0, C]$. The cost of the $\mathrm{m}^{\text {th }}$ lowest-cost agent among $n$ agents is

$$
c_{m}=F^{-1}\left(\frac{m}{n}\right)
$$

and the average of the $m$ lowest-cost agents is

$$
\overline{c_{m}}=\frac{n}{m} \int_{0}^{c_{m}} c f(c) d c
$$

Let us examine three specific cost distributions: uniform, triangular, and truncated normal. If the agents' costs are distributed uniformly on $[0, C]$, then $c_{m}=m C / n, \overline{c_{m}}=m C /(2 n)$, and $\rho=\mathrm{m} /$ $(2(m+1))$. Except for very small $m$, the dissipation ratio is close to $1 / 2$. Thus, the agents dissipate half of the prize value when there are many prizes and less than half only when the prizes are very few.

If the agents' costs are distributed on $[0, C]$ according to a symmetrical triangular distribution centered at $\mathrm{C} / 2$, then (29), (30) and (27) are given by

$$
\begin{aligned}
& c_{m}=\left\{\begin{array}{cl}
C \sqrt{\frac{m}{2 n}}, & 2 m \leq n \\
C\left(1-\sqrt{\frac{n-m}{2 n}}\right), & 2 m>n
\end{array}\right. \\
& \overline{c_{m}}=\left\{\begin{array}{cl}
\frac{C}{3} \sqrt{\frac{2 m}{n}} & , 2 m \leq n \\
\frac{C}{6 m}(2(n-m) \sqrt{\mathrm{z}}-3 n+6 m) & , 2 m>n
\end{array}\right. \\
& \rho=\left\{\begin{array}{cc}
\frac{2}{3} \sqrt{\frac{m}{m+1}}, 2 m \leq n \\
\frac{2 \sqrt{z}(n-m)-3 n+6 m}{3 m(2-\sqrt{z-2 / n})}, 2 m>n
\end{array}\right.
\end{aligned}
$$

where $z \equiv 2(n-m) / n$. It can be shown that (33) has a unique maximum of $2 / 3$ when $2 m=n$. Thus, the

8) Since there are $n$ agents, it is proper to define $f()$ as a discrete probability mass function. However, for analytical convenience, we will treat $f($ as a continuous density. 
most intense competition occurs when there is a prize for one out of every two agents and the agents then dissipate two-thirds of the value that they compete for.

If the agents' costs are distributed on $[0, C]$ according to a truncated normal distribution centered at $\mathrm{C} / 2$, we cannot obtain a closed-form solution but can confirm graphically that the results are qualitatively similar to the case of the triangular distribution. With $n=100$ agents, maximum cost $C=200$, and standard deviation of 40 , the dissipation ratio reaches a unique maximum of 0.69 when $m=37$. Unlike our earlier results with identical agents, the most intense competition with cost-heterogeneous agents occurs closer to the race extreme, with prizes for only $37 \%$ of the agents. Figure 12 plots the dissipation ratio (27) as a function of the number of prizes for the three cost distributions.

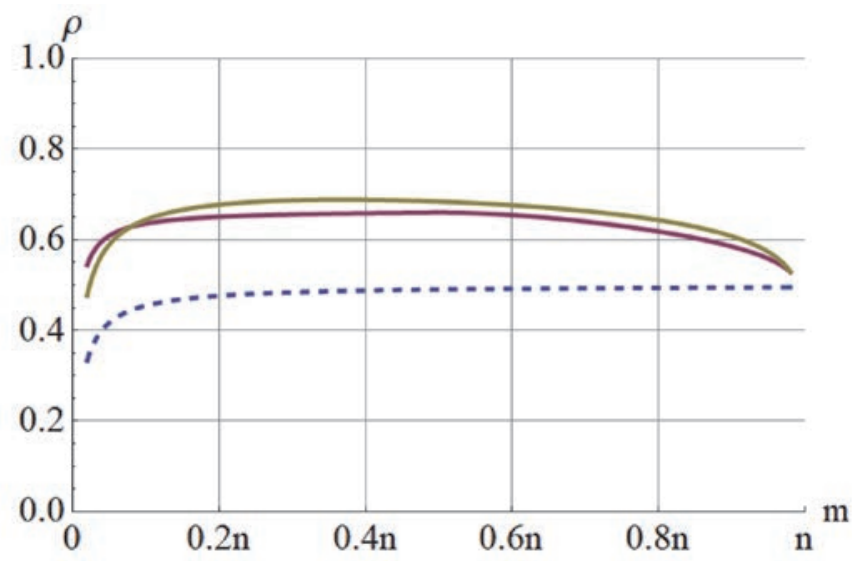

Figure 12. Fraction of aggregate value dissipated as a function of the number prizes if agents' costs are distributed on [O, C] according to a uniform distribution (dashed curve), triangular distribution (top curve), and truncated normal distribution (middle curve). The plots were drawn using $C=200, n=100$, mean 100 , and the standard deviation of the normal distribution set at 40 .

\section{Conclusion}

We used multi-prize contest theory to study the connection between intensity of competitive effort and the relative numbers of winners and losers. We found that identical agents make the most effort in contests that have more winners than losers. That is, the kind of competition that intensifies competitive striving among identical agents lies closer to the musical-chairs extreme than to the race extreme. However, if agents differ in the cost of effort, then the most intense competition occurs when the number of prizes is between two-thirds and half of the number of agents, i.e., closer but not too close to the race extreme. Agents make less competitive effort in contests that allow very few winners or losers, presumably because the probability of ending up as a rare winner or a rare loser is low. These results require verification and qualification through more realistic models and simulations with agents who are heterogeneous in terms of preferences, endowment, cost of effort, and risk aversion. Economic 
experiments and comparative case studies of actual competitions are also necessary to better understand the relationship between the intensity of competition and the relative numbers of winners and losers.

\section{References}

Blascovic, J. (2008) "Challenge and threat" in A. J. Elliot (Ed.), Handbook of Approach and Avoidance Motivation, Psychology Press, New York, 431 - 445

Clark, D.J., and Riis, C. (1996) “A multi-winner nested rent-seeking contest” Public Choice 87, 177-184

Elliot, A. J. (2008) "Approach and avoidance motivation" in A. J. Elliot (Ed.), Handbook of Approach and Avoidance Motivation, Psychology Press, New York, 3-14

Hirsch, F. (1977) The Social Limits to Growth, Routledge \& Kegan Paul, London

Konrad, K. A. (2009) Strategy and Dynamics in Contests, Oxford University Press, Oxford

Siegel, R. (2009) “All-pay contests” Econometrica 77:1, 71-92 